\title{
Quadro normativo per la Geotermia a Bassa Entalpia
}

Roberto Spalvieri

Coord. Commissione Geotermia Ordine Geologi Lazio robertospalvieri@geologilazio.it

\author{
Marco Vinci \\ Commissione Geotermia Ordine Geologi Lazio \\ marcovinci@geostudiovinci.com
}

Le nuove direttive comunitarie in materia di utilizzo di energie rinnovabili stanno lentamente offrendo spazio all'utilizzo della risorsa geotermica che, se pur non diffusamente distribuita sul territorio nazionale, trova in alcune Regioni un ambito applicativo preponderante. Va comunque ben distinta la geotermia per la produzione energetica (a media e alta entalpia) da quella dedicata alla "climatizzazione" degli edifici, che differisce in termini sostanziali per i suoi criteri di base. La diffusione sul territorio nazionale della tecnologia definita geotermia a bassa entalpia o geoscambio risulta ancora limitata per via della scarsa conoscenza dei principi applicativi da parte di molti professionisti e delle corrette modalità tecnicooperative da parte della gran parte degli installatori, nonché fortemente condizionata da una profonda carenza normativa a carattere nazionale ed ancor più a livello regionale. In un panorama mondiale complesso, con un vincolo chiaro imposto dal protocollo di Kyoto che definisce l'obiettivo al 2020 della diminuzione delle emissioni di $\mathrm{CO}_{2}$ in atmosfera del $6,5 \%$ rispetto al 1990 e con una comunità scientifica internazionale concorde sugli effetti negativi derivanti dall'emissione di gas serra e polveri sottili derivanti dall'uso di combustibili, l'Unione Europea promuove la diffusione di tecnologie che non prevedano l'utilizzo di fonti di energia fossile, con particolare riferimento al settore dell'edilizia. La direttiva 2002/91/CE sul rendimento energetico dell'edilizia ed il documento strategico del 10 gennaio 2007, definiscono chiaramente la strada da seguire per una corretta politica energetica europea, che chiede l'utilizzo di fonti energetiche rinnovabili e la crescita dell'efficienza energetica. Nel complesso panorama comunitario, solo alcuni paesi si sono dotati di uno strumento normativo efficace. In Italia la situazione è decisamente complessa e frammentaria. Lapplicazione dei sistemi di geoscambio per la climatizzazione rientra di diritto tra le tecnologie che permettono di ottenere un miglioramento del rendimento energetico, se pure nelle norme specifiche non sia espressamente menzionata (D.Lgs 19 agosto 2005, n. 192 corretto e integrato con il D.Lgs. 29 dicembre 2006, n. 311).

A livello nazionale la geotermia è normata già a partire dal R.D. 27 luglio 1927 n.1443, successivamente dalla Legge 9 dicembre 1986, n. 896; bisognerà attendere però la Legge 23 luglio 2009 n. 99 (Art. 27 comma 28) per l'emanazione di un regolamento finalizzato al riassetto della normativa in materia di ricerca e coltivazione delle risorse geotermiche e sentir parlare concretamente di media e bassa entalpia. L'Art. 27 comma 39 della suddetta Legge definisce che entro sei mesi dalla sua data di entrata in vigore, il Ministro dello sviluppo economico, di concerto con il Ministro dell'ambiente e con il Ministro delle infrastrutture e trasporti, di intesa con la
Conferenza unificata di cui all'articolo 8 del decreto legislativo 28 agosto 1997, n. 281, emani un decreto volto a definire le prescrizioni relative alla posa in opera degli impianti di produzione di calore da risorsa geotermica, ovvero sonde geotermiche, destinati al riscaldamento e alla climatizzazione di edifici, per cui è necessaria la sola dichiarazione d'inizio attività. Analoga dicitura verrà riportata nel Decreto legislativo n. 28 del 3 marzo 2011, all'Art. 7- comma 4, ma ad oggi siamo ancora in attesa di un decreto attuativo nazionale che derima i dubbi in termini di regimi autorizzativi.

Sarà il D.Lgs. 11 febbraio 2010 n. 22, art. 10, a definire nell'ambito delle applicazioni geotermiche inequivocabilmente tra le piccole utilizzazioni locali di calore geotermico, quelle effettuate tramite l'installazione di sonde geotermiche che scambiano calore con il sottosuolo senza effettuare il prelievoreimmissione nel sottosuolo di acque calde o fluidi geotermici. Il medesimo strumento normativo chiarisce che sono le Regioni, o Enti da esse delegate, le autorità competenti per le funzioni amministrative e di vigilanza per queste utilizzazioni locali geotermiche. Sempre alle Regioni è affidato per tale tipo di applicazioni la definizione di una specifica disciplina applicativa, con previsione di procedure semplificate.

Allo stato attuale, pertanto, rimane attesa l'emanazione del Decreto Ministeriale Attuativo del citato D.Lgs. 28/2011 che dovrà indicare le prescrizioni per la posa in opera degli impianti di produzione di calore da risorsa geotermica, ovvero sonde geotermiche, destinati al riscaldamento e alla climatizzazione di edifici, e individuare $\mathrm{i}$ casi in cui si applica la procedura abilitativa semplificata (PAS) di cui all'art. 6 del Dlgs. n. 28/2011. Altrettanto attesa resta l'attivazione del $\mathrm{I}^{\circ}$ Conto Energia Termica che garantirà l'incentivazione degli interventi per la produzione di energia termica e di quelli sull'efficienza energetica. L'incentivo sarà commisurato all'energia prodotta e al risparmio energetico realizzato, secondo tabelle indicanti i valori in base alla taglia degli impianti e alla zona climatica di pertinenza. Per gli impianti a biomassa o a Pompe di Calore fino a $35 \mathrm{~kW}$, come per quelli solari termici fino a $50 \mathrm{mq}$, gli incentivi saranno costanti e forfettari per la durata di due anni. Per gli impianti più grandi, invece, l'incentivo sempre costante e forfettario durerà cinque anni.

In funzione di quanto riportato nel Piano di Azione Nazionale (PAN), previsto dalla direttiva RES 2009/28/CE quale documento programmatico che fornisce indicazioni dettagliate sulle azioni da porre in atto nell'uso dell'energia da fonti rinnovabili (all'Italia è stato assegnato l'obiettivo 2020 di coprire con le FER il 17\% dell'energia prodotta e dei consumi lordi nazionali), è stato emanato il D.M. Sviluppo economico del 15 marzo 2012. Tale decreto, noto come 
Burden Sharing, definisce e quantifica gli obiettivi intermedi e finali che ciascuna Regione e P. a. deve conseguire ai fini del raggiungimento degli obiettivi previsti dal PAN fino al 2020, in materia di quota complessiva di energie da fonti rinnovabili (FER Elettriche+FER Termiche+biogas) e sul consumo finale lordo di energia CFL (elettricità+ riscaldamento/ raffreddamento+trasporto).

Nonostante ciò, solo alcune Regioni hanno definito normative, regolamenti o linee finalizzate alla regolamentazione della geotermia a bassa entalpia. Per le Regioni più virtuose si sono poi definiti regolamenti attuativi provinciali come quelli emanati dalle Provincie di Milano, Bergamo, Como, Lecco, Brescia, Pavia, Mantova, Sondrio, Varese, Biella, Vicenza, Treviso, Trento e Bolzano.

La Regione che per prima si è dotata di uno strumento normativo articolato è stata la Lombardia che già con il Regolamento Regionale 24 marzo 2006 n. 2 nel suo Art.32 ha disciplinato l'uso delle acque superficiali e sotterranee, per gli impianti di geoscambio a ciclo aperto (pompaggio e reimmissione noti anche come open loop). Sempre nel 2006 la Regione Lombardia con la Legge 11 dicembre 2006 n.24 (art.10) promuove l'utilizzo delle risorse geotermiche a bassa entalpia e l'adozione di procedure semplificate per l'installazione e la gestione di sonde geotermiche. Con questa Legge l'installazione nel sottosuolo di sonde geotermiche che non comportano il prelievo di acqua è libera o vincolata dal rilascio autorizzativo da parte della Provincia territorialmente competente e sottoposta alla procedura di registrazione dell'impianto in apposita banca dati regionale (RSG). Nel febbraio 2010 la Regione Lombardia emana in attuazione dell'art. 10 della L.R.11 dicembre 2006 n. 24 il Regolamento Regionale 15 febbraio 2010, n. 7 per l'installazione di sonde geotermiche che non comportano il prelievo di acqua (closed loop), disciplinando modalità tecnico-operative per l'installazione e la gestione degli impianti, caratteristiche minime dei relativi progetti, criteri tecnici, geologici e territoriali in base ai quali è rilasciata l'autorizzazione per l'installazione di sonde geotermiche, profondità di perforazione e installazione delle sonde geotermiche nonché i limiti al di sotto dei quali è richiesta l'autorizzazione provinciale, criteri più restrittivi per assicurare il rispetto dell'ambiente, requisiti e modalità per la certificazione di qualità delle imprese operanti nel settore della perforazione e installazione delle sonde geotermiche, nonché dei controlli a carico delle imprese installatrici per il mantenimento della certificazione di qualità. L'iter normativo intrapreso dalla Regione Lombardia rappresenta in termini assoluti il più completo ed evoluto quadro nel panorama nazionale.

La Regione Piemonte ha seguito un iter normativo diverso, partendo con la L.R. 22/1996 che ha definito i principi per l'uso e la tutela delle acque sotterranee, prevedendo specifici vincoli di tutela delle falde profonde. Da questo ha emanato criteri e linee guida relativi ai sistemi a ciclo aperto (open loop). Attualmente non esiste una normativa specifica per i sistemi a sonda geotermica (closed loop), fatta eccezione per il vincolo di utilizzare pompe di calore con COP minimo pari a 4 (Allegato 4 dello Stralcio di Piano per il riscaldamento ambientale e condizionamento dell'11 gennaio 2007).

La Regione Toscana con la L.R. 39/2005 ha stabilito le procedure amministrative per l'istallazione delle sonde geotermiche, specificando che qualora lo scambio termico avvenisse senza prelievo di fluidi, l'iter autorizzativo segue la procedura semplificata dell'Autorizzazione Unica (descritta all'Art.11 della medesima legge).

La Regione Valle d'Aosta ha di recente emanato la L.R. $1^{\circ}$ agosto 2012, n. 26 che di fatto detta le disposizioni in materia di interventi regionali per la promozione dell'uso razionale di energia, con particolare riguardo alle energie da fonte rinnovabile, senza entrare nello specifico delle applicazioni geotermiche.

La Regione Veneto già con il Piano di Tutela delle Acque approvato con deliberazione del Consiglio regionale n.107 del 5 novembre 2009, all'art. 31, comma 3, stabilisce che, ai fini della protezione delle acque sotterranee, la realizzazione di sistemi di scambio termico con il sottosuolo che non prevedano movimentazione di acqua di falda (closed loop) è autorizzata dalle Provincie. Queste ultime hanno attuato appositi regolamenti e linee guida: già a partire dal 2009 (Treviso), nel 2011 (Venezia, Rovigo, Vicenza), nel 2012 (Verona) o sono in via di definizione (Padova, Belluno).

La Regione Friuli Venezia Giulia per le piccole utilizzazioni geotermiche che scambiano calore direttamente con il sottosuolo o con le falde ospitate negli acquiferi senza effettuare il prelievo di fluidi, prevede attraverso il Piano di Tutela delle Acque, ai fini della protezione delle acque sotterranee, un autorizzazione provinciale.

Nel Trentino Alto Adige la Provincia Autonoma di Bolzano con DGP n³564 del 26 settembre 2005 ha definito specifici obblighi prima e durante la realizzazione dei sistemi di geoscambio, inserendo anche direttive tecniche per esecuzione in sicurezza delle perforazioni, finalizzate alla tutela delle falde. La Provincia Autonoma di Trento con l'Art. 30 bis del DGP 26 gennaio 1987 n. 1-41/Legisl., prevede che l'installazione di sonde geotermiche sia soggetta ad autorizzazione del servizio Geologico della Provincia Autonoma di Trento (e parere dell' Agenzia Provinciale per la Protezione dell'Ambiente APPA).

Nella Regione Umbria con Delibera di Giunta Regionale n³86 del 08/03/2010 sono state dettate le indicazioni tecnico-amministrative per la realizzazione di pozzi per lo sfruttamento di risorse geotermiche a bassa entalpia.

Il panorama normativo delle restanti Regioni italiane vede lenti procedimenti istruttori in corso di definizione, a testimonianza di un complesso processo di regolamentazione territoriale che potrebbe ad oggi ostacolare l'effettiva affermazione della tecnologia o permettere il suo anticipato declino, dovuto ad interventi approssimativi, privi di qualità e non dimensionati al serbatoio geotermico dal quale ricevono alimentazione. A tal proposito, ben vengano le norme UNI sui progetti riguardanti il dimensionamento, l'installazione e i requisiti ambientali dei Sistemi geotermici a bassa entalpia basati sul principio della pompa di calore, entrati dal 09 maggio 2012 nella fase di studio nell'inchiesta pubblica finale per l'emanazione delle specifiche normative. 